\title{
Sociosemiotics and Metalanguage: The Case of Translanguaging
}

\author{
Anti Randviir \\ Department of Semiotics, University of Tartu, Estonia.
}

\begin{abstract}
The age of transdiscipinarity has brought along the fading of boundaries between disciplines. This has engaged both the fusion of metalanguages that are used for the description of cultures and sign-processes, and the launch of novel proposals for metalinguistic vocabularies. In the case of the study of culture, communication and sign-processes, it is natural that all such developments are connected with the paradigm of semiotics. At times the relevant metalinguistic and methodological proposals challenge traditional semiotic vocabulary, methods and methodological truths. This has brought along the need to recall the semiotic roots of the study of semiosis and communication, and to review transdisciplinary metalanguage in order to avoid possible misinterpretations or unnecessary repetitions of the established agreements in the paradigm of semiotics as a possible ground for transdisciplinary study of human interaction and cultural processes. However, besides occasional theoretical confusion, the spread of the semiotic methodology and vocabulary across applied scholarship has enriched semiotics in its faculties of field studies.
\end{abstract}

Keywords: sociosemiotics, metalanguage, transdisciplinarity, modality, multimodality, mediality, coding, semiosis, modelling, translanguaging.

Contemporary scholarship has, both in the domain of humanities and social sciences, as also in the so-called hard sciences, passed a fairly clear transition from monodisciplinary studies towards transdisciplinarity. Between the two there have been developmental stages like cross-, poly-, multi-, and interdisciplinary studies. Ideas about binding and combinining disciplines and eventually dropping disciplinary boundaries have been rooted in understanding scholarly analysis as departing not from narrow paradigmatic principles, but from objects of research themselves. This means that the analysis of cultural, societal, or natural phenomena departs from necessities, not from avalabilities of taking diverse viewpoints at them. Transdisciplinary studies have an object-centred essence, and can apply toolkits from an unlimited number of disciplines. It is worthwhile to keep in mind that such understanding of conducting scholarship is not a novel thing, and dates back way longer than the second half of the twentieth century, to the time when several publications, associations and other institutions for interdisciplinary studies were founded. Secondly, it is useful to recall the proximity of interdisciplinary studies and interdisciplinary education as proposed by American pragmatism. The semiotic context of the latter points at an extremely noteworthy effort to gather all science under one umbrella at the beginning of the twentieth century. Of course, that was the enterprise of International Encyclopedia of Unified Science introduced in 1938 by O. Neurath, N. Bohr, J. Dewey, B. Russell, R. Carnap, and C. W. Morris. It was probably neither by chance nor because of the editorial role of C. W. Morris what led to selecting semiotics as the first main principal and methodological way of theorising about things in the universe (Morris 1938).

Semiotics, alongside with its methods and vocabulary, quests about its status either as a method or an individual paradigm of thought, has been and probably will be the most favourable candidate for basing transdisciplinary studies. The nature of human communication and the seeming universality and understandability of the semiotic vocabulary used to describe it makes 
semiotics and semiotic language a very desirable bride to marry or at least to borrow. Naturally, semiotic terms for units and processes of communication are essential for the description of any interaction. That makes them oftentimes seemingly self-explanatory and subject for mundane understanding, rooted in the social stock of knowledge and in God's Truth. At the same time, this universal presence of semiotic units (for the latter see importantly Eco 1976, 62-68) and processes frequently triggers attempts to re-disclose the contents of the descriptive terms in diverse and numerous metalanguages. In these enterprises the actual semiotic essence, and moreover the semiotic disciplinary background of these terms, remains unnoticed or very superficially touched at best. From the viewpoint of semiotics, the backbone of semiosic units and processes is extended and even bent every so often that the metadiscursive multiplicity of vocabulary has led to oblivion in recognising the actual objects of analysis. Thus-transdisciplinary use of basic semiotic notions can be seen in contradiction with the heuristic value of targeting that bubbled metalanguage.

Weirdly, the essence of studying communication and sign creation has not lead only to confusing transformations of the semiotic metalanguage in diverse other disciplines, but concerns the definition of semiotic research itself as well. Illustrations can be brought by numerous quite specialised branches of semiotics that are sometimes dedicated to very minute research objects (advertising, media, literature, cinema, etc.). On the other hand, the past decades have naturalised such larger fields of study as cultural semiotics, biosemiotics, sociosemiotics. Regarding the latter, a trial of demarcating its boundaries can be based on remembering the three fundamental dimensions of the semiotic research as established by C. Morris (Morris 1971, 43-54). Neigbouring the syntactic and the semantic dimensions, it is the pragmatic angle that has narrowed the introduction of social semiotics and sociosemiotics largely to the re-invention of the wheel (see Cobley, Randviir 2009), but apparenly recurrent calls for the sources of semiotic studies may sometimes occur useful. The pragmatic aspect of semiotic research obviously must focus on the techniques, operations functions, and strategies of communication and semiosis that people undertake and go through in interaction with the purpose of effective exchange of messages and meanings. Interestingly, but not very fortunately, here we can find another puzzle that has to do with semiotics, the semiotic vocabulary, and especially with the so-to-speak sociosemiotic issues in the age of transdisciplinarity. Namely, certain matters concerning pragmatics have been developed into individual research subjects that seemingly stand separately from the syntactic and the semantic field which, according to Morris cited, is a logical impossibility as such. Furthermore, the operational side of those pragmatic processes and procedures have sometimes been associated with the field of sociosemiotics, whilst it is inconceivable to interpret the latter field as independent from cultural semiotics or biosemiotics. As the topic concerns overall transdiciplinary relations, especially in terms of vocabularies, and also relations between different fields inside semiotics itself (specifically general semiotics, theoretical semiotics, applied semiotics, sociosemiotics, cognitive semiotics), it seems worthwhile to stop at some recent exaples of terminological and disciplinary entanglement that have particular actuality for sociosemiotics.

Let us try to interpret, in the light of the above, the book Making Signs, Translanguaging Ethnographies: Exploring Urban, Rural and Educational Spaces (edited by Ari Sherris, Elisabetta Adami) published in 2019 that takes up the topic of translanguaging in the more general frame of transdisciplinarity whilst the latter is viewed largely through the prism of social semiotics. The book itself contains practical case studies, theoretical and to a some extent metatheoretical parts in formal, less formal, and informal modalities. It begins with clarification of theoretical perspectives by J. Bezemer and G. Kress, and A. Sherris and E. Adami. The reader is then offered a most interesting "Bricolage of Ideas" in the form of opinions of basic theoretical issues by J. Blommaert, O. Garcia, G. Kress and D. Larsen-Freeman. These ten theoretical issues have been raised by $\mathrm{E}$. Adami and A. Sherris, who also offer their comments as a conclusion to this part of the publication. The book then continues with case studies carried out and discussed by E. Adami, A. Archer and A. Björkvall, F. Banda, H. Jimaima and L. Mokwena, J. Bradley and E. Moore, N. Perera, S. Goodchild and M. Weidl, and A. Sherris, P. Schaefer and S. M. Aworo. These practical studies are further seen by A. Sherris and E. Adami from the viewpoint of complexity theory and social semiotics in their "Heterarchic Commentaries" that conclude the publication.

It is necessary to notice that the book is organised very nicely in the form of discussion, while that discussion holds also for individual articles dedicated to the analysis of diverse localities and phenomena. On the other hand, the opening discussion in the "Bricolage of Ideas" also seems to explain some theoretical troubles with both the following case studies and the overall bias of the book, as with translanguaging in general. Namely, the biggest obstacle concerning both the theoretical and practical analytic stands in the book is that language and linguistics, sociolinguistics and sociology of language have set such a conceptual cage for the authors that the only tool they eventually grab to file the bars of that cage is modality, added by occasional use of multimodality. This is why oftentimes an impression is left as if the actual phenomena and contexts of communication that the authors try to vivisect, remain on the other side of the 'iron cage'. Let me try to explain this briefly, centering at the metalinguistic issues of translanguaging, and still valuing highly the case studies carried out under its label. 


\section{MODALITY AND MEDIALITY}

Modality, as a matter of fact, is what the book is largely about all in all, and this influences also understanding the paradigm of translanguaging. Modality is amongst one of the central notions at the description of sign-making and communication, and it naturally has been a subject of vast and long academic discussions in a number of study domains and from multiple viewpoints. In our context, modality as having to do, by and large, with 'the mode of expression' has been tied to the issue of mediality. This means that the original psychological and purely semiotic faculties have been paralleled, if not crossed with more technical matters. Such a stand is in an obvious disagmeement with classical semiotic understanding presented by, for example, F. de Saussure who discriminated very clearly between the psychological, physiological, and physical levels of communication as the ones defining the study area of his semiology (Saussure 1959,12 ). Modality as bound with mediality means that we are dealing with the analysis of bundles of and in communication where at least the following phenomena meet: signs, ways we use signs as defined by the accent or mode of expression, means of expression in terms of sign systems, and different media channels through which messages are transmitted. Therefore, modality and mediality are inevitably ambiguous terms. Obscurity rises when we realize that practically all human communication is subject to redundancy: a tendency to simultaneously use more sign systems than minimally necessary in order to convey the meaning intended. Thus, multimodality and multimediality come in as descriptive notions, alternative for the umbrella-term of redundancy-they allow synoptic analysis of those modes and media that are used in particular cases of communication, whereby the concept of redundancy, for example, seems to be left aside. The range of multimodal and multimedial analysis is quite immense, ranging from linguistic modalisation of discourse (exclamation, statement, parody, etc.) to more semiotic issues that eventually reach the levels of objectivity and subjectivity, truth and falsity (for a comprehensive overview of multimodal studies see Kress 2001). Those semiotic issues are bound with mediality, for they have to do with the channelling of messages, beginning from choosing between or combining verbal, gestural, pictorial and other discourses. Messages are (a) transmitted in diverse media; (b) translated from one media into another; they are (c) encoded in several types of discourse (also synchronically); (d) encoded in several sign systems; (e) encoded in several versions of a specific type of a sign system (as in the case of pidgin). Obviously, the multiplicity that holds for encoding messages both in modality and mediality must concern also the side of the addressee and the relevant processes of decoding. Overlap in the mutual competence and understanding of the supposedly correct practises of coding should principally be at least some guarantee for successful transmission of messages as also for the adequacy of messages in terms of their connection with the reality (at this moment, it does not matter what is kept in mind under that notion). Multimodality and multimediality should thus be responsible also for the truthfulness of messages. The truth value of messages seems then to depend on the specific nature of the reality and phenomena reported (physical, social, virtual, objective, subjective or other possible realities) as also on the coding abilities of communicative partners. Perhaps not too surprisingly, the extremely manifold issues that multimodality and multimediality are responsible for may eventually result in specific quite paradoxic eventualities (which, in the end, are not that paradoxic altogether) as, for example, the possibility of the co-presence of telling the truth and lying at the same time. This can be the case at bringing out the truth through irony, as one of the simplest illustrations.

The case studies described in the book under inspection are so much biased towards issues pertaining to modality, multimodality and multimediality (at times also to transmediality) that frequently the original context and meaning of translanguaging as it appeared in educational and sociocultural linguistics (see e.g. Lewis et al. 2012) is lost. Instead, translanguaging is associated and explained through the following terms and concepts: polylanguaging, metrolingualism, linguistic landscaping, multimodal translanguaging, sign-making, translocality, translocal meanings, transcontextuality, remediation, repurposing, recontextualization, signage, plurilingual practices, codemeshing, superdiversity. Although notions like the mentioned have become characteristic of the particular age of transdisciplinarity we are living through, there are a few fundamental issues to keep in mind when using them. First of all, between most of them there holds the relation of tautology: they are mutually explanatory. Secondly, most, if not all of them, have to do with (multi)modality and (multi)mediality. Thirdly, and perhaps most importantly-they all belong to the paradigm of semiotics as the latter aims at the description of semiosis or the sign-creation process in its multiple facets and aspects. In short, it has been commonplace in the very long history of semiotics or the study of semiosis and signs, to understand meaning-making as a social process that takes place in the diverse dimensions of the environment (geographical, natural, cultural, economical, political, linguistic, etc.) by the use of shared codes that are applied to encoding and decoding messages that can emerge simultaneously in different forms and can be constructed by the simultaneous use of a variety of sign systems. Successful meaning-making and exchange of messages depend very largely on the competence (linguistic, communicative, cultural, semiotic competence) of communicators, and should therefore engage all the multimodal and multimedial capacities of them. J. Bezemer and G. Kress introduce the reader to the fact that "multimodality invites linguists to explore how speech and writing each combines with other modes" (Bezemer, Kress 2019, xv), and propose that "ii]nstead of exploring the use of speech alone, 
the multimodalist might also consider how vendor and customer negotiate prices or pass objects using combinations of gaze, gesture, speech and movement of their bodies. Without attention to all the semiotic resources in play, accounts of sign-making cannot be satisfactory" (Bezemer, Kress 2019, xv-xvi).

It is hard not to agree with this opinion. Yet it is an absolute imperative to remember that suchlike approach to communication and meaning-making has been not only valued, but also applied in the paradigm of semiotics. This comes from the multidimensional nature of communicative situations that holds for the object level. That multidimensionality has to do with two parametres at least: a) the multitude of contextual issues, and b) the multitude of sign systems used to transfer messages. Apparently, what is rather at stake in the framework of this book, has to do with the simultaneous application of very diverse sign systems in order to communicate messages with intended meaning(s).

\section{REDUNDANCY AND CODING}

The phenomenon of multidimensionality of semiosis brings along another field of issues that has been treated in semiotics and its adjacent disciplines under the notion of redundancy which, by the most general definition, is a term to refer to certain techniques used to make the predictability of intended decoding of messages higher. In relation to our topic, and to the issue of translanguaging in general, it is hereby appropriate to recall a part of J. Fiske's encyclopaedic explanation of redundancy:

"It helps overcome problems associated with the audience: the encoder will build more redundancy into the message if $s /$ he predicts that the audience is not strongly motivated towards what s/he has to say, or if $\mathrm{s} /$ he knows that the message is aimed at a large, heterogeneous audience with a variety of backgrounds and motivations. Thus [...] an ad for a chocolate bar is more redundant than a technical ad in a specialist journal." (Fiske 1994, 260).

Redundancy thus involves several techniques of overcoding (see Eco 1976, 133-135), and presumes that partners of communication share understanding of the communicative context as certain transformations that happen to both the semiotic units and sign systems in unexpected associations of changed contexts. In those transformed contexts and semiotic realities (including also sign systems and semiotic units), overcoding ensures that communicative partners are able to apply specific correction techniques to what would seem as aberrant encoding of messages at first glance. Clearly, these are topics re-introduced in the paradigm of translanguaging under the notions of 'recontextualisation' and similar glamorous terms already mentioned. It is hereby very important to remind a crucial and beneficial understanding how to treat different encoding strategies in principally dissimilar communicative situations. Namely one ought to think back to B.
Bernstein's distinction between restricted and elaborated codes as connected with the different linguistic, social, and cultural background of communicators and audiences. Although Bernstein departs from seeing these two principally distinct types of codes as connected with the social background of speakers, the topic is semiotically much deeper, and bypasses the limits of oral behaviour and the universe of stricktly natural language. Besides calling elaborated and restricted codes (and subclasses of them) ideal types or ideal cases (Bernstein 1964, 58), Bernstein stresses the importance of extraverbal signals and messages that enhance the arrival of the intended meaning to the addressee, especially in the case of the prevalence of the use of restricted codes. Extraverbal channels like "expressive associates of associates of the words (intonation, etc.), messages transmitted through gesture, physical set, and facial modifications" (Bernstein 1964,58 ) apply more to restricted codes and help to enlarge the share of the contextual associations of communication between the communicators.

Clearly, this is exactly the case which is dealt with under the faculty of contextualisation, recontextualisation, plurilingual practices and other notions in the paradigm called translanguaging. Eventually, redundancy and the mutual support between sign systems with the goal of trying to assure the arrival of the message in its intended content seem to be assisted also by semiotic units as the latter can include individual meaningful units from the level of signs to the levels of texts and culture topics. This is important, for interaction between larger meaningful units, as text is a factor to simultaneously make a semiotic space-or the semiosphere-more heterogenous and also even to enlarge the amount of psychological, cultural, semiotic, social ties between structures and agents in semiotic spaces. The context-dependance of semiotic units applies both to elaborated and restricted codes. In the case of elaborated codes, semiotic units are activated by the help of the abstract core of signs, and that abstract essence makes it possible to encode meanings and messages according to the specificities of the context. In the case of restricted codes, meaningful units are founded on the shared past context(s) of communicators, and meanings are activated exactly on the grounds of mutual interdependent contextualisation of meaningful units as it holds between the addressant and the audience.

\section{SEMIOTIC UNITS AND MATERIALITY}

It seems that this difference in understanding the contextuality of semiotic units either in abstract or concrete manner lies at the foundation stones of problems surrounding the paradigm of translanguaging. We can see this in several paragraphs of the "Bricolage..." chapter of the book. Let us consider, for example, some of the following statements. When J. Blommaert admits that "I have replaced 'meaning' almost entirely by 'effect" and that the "recognizability [of meaning-as-effect] is usually not 
a feature of the resources we use alone, but more of the practices we deploy them in" (Blommaert et al. 2019, 12), then this may lead to very serious misunderstanding of the essence of the semiotic units from the viewpoint of semiotics. This is seconded by G. Kress who takes such an empirical view on meaning to even more materialistic level of understanding. Kress claims: "In the semiotic work of sign-making, signifieds ('elements of significance') are joined with signifiers (material means apt for making signifieds evident)" (Blommaert et al. 2019, 12). These statements are in harsh contradiction with the grounding standpoints of contemporary semiotics, both from the Peircean and the Saussurean styles of theorizing. On the one hand, the replacement of meaning with effect is an open highway for driving away from the semiotic understanding of meaningful behaviour to hard behaviourism against we have been warned (e.g. Nauta 1972 , 42) and have been provided by C. S. Peirce with the notion of interpretant. Practically, the same was the logic of F. de Saussure when he was talking about signs as arbitrary unions of sound-images and concepts. Hereby it is probably not out of place to make yet another warning call to remind that Saussure's sound-image is not comparable to the signifier, as is not his concept comparable to the signified (see Saussure 1959, 65-70). Such misinterpretations come directly from convinient mistranslations that, instead of a possibly popularizing intent have but confused the whole Saussurean legacy and the image of the semiological paradigm. Even as associations of sound-images and concepts, signs are not necessarily the "material realization of socially significant phenomena" as Kress claims them to be (Blommaert et al. 2019, 12). The material ontology of signs is only a possible parameter for them-this is the essence of semiotic units as definable through their meaning-ful character. The most influential consequence of such unnecessary and, in fact, semiotically inadequate comprehension of meaningful units as approachable directly through overt behaviour and through their materiality seems to have been fatal for the present book under inspection, as for at least some basic stands of the translanguaging thought as such. Namely: cognitive processes are not necessarily revealed in overt behaviour, not in interaction, not in advertisement plates, not in street signs. In this sense, Kress' suggestion that "the fundamental principle of human sign-making is that the signifier (a material element) is an apt means of making the signified (an element of significance for the sign-maker) materially evident" (Blommaert et al. 2019, 14) must be taken with extreme caution. It is clear that the socialness of meanings may lay in things, but the socialness of things does not imply that meanings can be directly approached by materiality (including overt behaviour).

One of the central ideas of translanguaging theorizing seems unfortunately to accept and depart from taking semiotic units as if not material, then at least largely definable through the material dimension and properties. Interestingly, this sets this paradigm into the permanent state of an internal contradiction. If the significance of signs could be found in their material properties, then it ought to be logical that individual semiotic units as items could indeed be subject to the various operations referred to, in the translanguaging vocabulary, as 'translocalization' and other elegant notions mentioned at the beginning. By this understanding, sign-vehicles could simply be transported into different locations and contexts, and activated there by triggering off (for example, through education or instruction) the connection between what, in this paradigm, is comprehended by the signifier and the signified. However, translanguaging bases itself extensively on multimodal research that:

"is prompted by the fading away of previously (relatively) stable social arrangements. They had produced a degree of certainty and predictability about meaning-making, and perhaps made it possible not to attend to all the semiotic means involved" (Bezemer, Kress 2019, xvi).

On the one hand, multimodality is present at practically all communication and exchange of messages. In this sense, messages are-at least preferably-always overcoded to a certain extent, because this enhances them to be interpreted according to the intention of the sender.

\section{MULTIMODODALITY AND PLURIDIMENSIONALITY OF SEMIOTIC UNITS}

The inclination to interpret signs as material entities seems to be one of the reasons for translanguaging to see its research field in the translation of existing semiotic forms (sign-vehicles) into some other semiotic forms, possibly in secondary contexts. This can involve translation between natural languages or translation between different semiotic systems. Whilst these topics have been canonically treated through the notion of redundancy, in more modern language they have been approached through 'mode' and 'modality'. Characteristically, G. Kress sees 'mode as technology of transcription' (Kress 2010, 96-102) where:

"Mode is a socially shaped and culturally given semiotic resource for making meaning. Image, writing, layout, music, gesture, speech, moving image, soundtrack and 3 objects are examples of modes [...]" (Kress 2010, 79).

It is obvious that in the case of speech and writing we are dealing with two different ways of encoding natural language into messages, and that we can call speech and writing to be both modes and mediums of presenting meanings in specific forms of messages. Also, layout for presenting visual messages ought to be seen as a way of orgnising discourse in a preferred manner so as to maximise the possibility of connecting with the target audience. In this sense, layout as a way of organising discourse pertains also to other sign systems than merely the visual. The problem that arises here is evident: even if 'mode' is interpreted technically, its meaning involves already the presentation of messages in different sign systems, and also the specific organisation of discourse in the sign system selected. Multimodality 
which is one of the cornerstones of translanguaging thus becomes vague already at the application of 'mode' at its very roots, remaining yet on the level of the material interpretation of messages and semiotic units. Problems deepen when we add media and mediality to mode and modality. It seems as if, for example, the concept of multimediality as referring to the application of more than one technical mediums for the presentation of discourse (e.g. classroom lectures) overlaps completely with multimodality as deriving from the interpretation of 'mode' as in the above citation from Kress. Mode, media, and medium thus get merged, and the research object of translanguaging is largely lost.

The technical aspects of modality (as also of mediality) can, by and large, be associated with the perceptual faculty of communicators (visual, aural, kinesthetic channels and abilities), but 'mode' also entails purposes and biases of interaction, therefore the topic of modality gets bound with that of intentionality. Furthermore: the mode of communication in terms of its intention can frequently be revealed by the mediality of discourse. For example, the presentation of a classical text of fiction in the form of a comic book or a short video may be intended as its popularization, parody or something else, and the result of interpretation will depend on its context. This means that multimodal and multimedial texts are oftentimes not only communicative, but also metacommunicative, and thus subject to intersemiotic and intersemiosic analysis. The peculiarity of the latter pair is that they can be solved through the toolkit of intersemiotic translation (see e.g. Torop 2000) that possesses distinct methodological devices of analysing extracoded texts in the multitude of (trans)medial environments (see e.g. Dusi 2015).

Intersemiosis and intersemiotic translation means, amongst other methodological issues, that messages are usually multilingual in the semiotic sense. On the other hand, semiotic units are multimodal as well: we can only conditionally distinguish between the indexic, iconic, and symbolic signs. Rather, the latter three are ideal types that are combined or even mixed in the real life Therefore it is a huge and unfair mistake to label any truly semiotic analysis of multimodal phenomena as assuming static relations either between signs, constituents of signs, codes (including natural language and further levels of codes), societal relations or other structures and agents in communicative situations. Semiotic units and phenomena are inherently dynamic-this comes from their non-material essence and is one of the foundation stones of semiotic studies, be the birth of the latter set to whatever historical period. In the regular semiotic vocabulary, natural language is based on human biological resources that shape the human Umwelt. Natural language, in turn, can be used to interpret several human semiotic systems as the gestural language, theatre, fashion and others as based on it-whether the latter are called secondary, tertiary or other order sign systems is irrelevant. Multimodality in terms of the simultaneous usage of sign systems is due to overcoding messages in multiple semiotic systems, whether in many secondary semiotic systems or engaging also natural language. We must not approach the topic from the view that "human beings select an assemblage of signs (linguistic and multimodal)" and that "these signs are sometimes linguistic (words, phonology, morphology) and sometimes multimodal (gestures, visuals, clothing, technology)" as suggested by O. Garcia (Blommaert et al. $2019,15)$. As already stressed, there are no specifically 'multimodal signs', for signs usually possess indexic, iconic and symbolic dimensions simultaneously. This goes also for signs of natural language which thus cannot be contrasted with those hypothetical 'multimodal signs'. Likewise, in terms of coding, vocabulary ('words'), phonology and morphology are applicable not only to natural language, but also to secondary sign systems as languages. There exist additional cases of interest in which we might ask about relations between natural language as the primary semiotic system, and gesture language (and other artificial languages up to cinema, fashion and so forth), and gestures as pertaining to the sphere connected with the perceptual reality of the Umwelt. In the framework of semiotics, relations between suchlike sign systems-including diverse operations with these relations and semiotic units themselves - can be quite precisely approached through the established methods of analysing translation, including also intersemiotic transposition, translation between languages and other semiotic resources and the similar (see Sonesson 2014).

\section{MODELLING CREATES DIFFERENCES}

Semiotic overcoding of messages compels to touch another key issue that has been treated as defining for translanguaging: heterarchy. Alhtough being heuristically quite vague, it is possible to infer that heterarchy is a notion to designate the plurality of things, agents, and semiotic systems in the world as principally equal with no predominant factors in terms of power over any other-the world is materially and relationally "an undifferentiated manifold" (Sherris, Adami 2019, 3). Heterarchy is seen in the "multiplicity and transconnections in knowledge that avoid any predetermined hierarchy" (Sherris, Adami 2019, 171). Although meeting the ideology of political correctness, it does not seem to apply to the actual study of semiotic phenomena. Of course, on the one hand, semiotics has always been a transdisciplinary mode of theorizing that draws together the multiplicity of methods associated with diverse 'official disciplines' as regarding the actual research object, and if felt as unavoidably necessary, we can call such an approach 'heterarchic'. On the other hand, viewing the issue from the side of the object field, the situation is different, but also calls for doubting the need of 'hetearchy', not to talk about the proposed novel paradigm of Complex Semiotic Heterarchic Theory. Of course, prior to meaningful discourse or the loading of physical units with semiotic values, there exists the mysterious external 
world that is sometimes called the 'objective reality'. That serves as a pool of semiotic potentiality indicated by Peirce as the basis of Firstness (CP 1.300). However, when already engaged with the semiotic world filled with texts, smaller semiotic units and codes, talking about the equality of, for example, units of discourse, is a semiotic impossibility. While paradigmatic relations between meaningful units can, at a certain hypothetical elementary level, be viewed as equal, then on the level of semantic fields those relations are already laid on a certain ground, that is-potentially equal meaningful units, for example connotations, are centered around a more specified unit of intention, a denotation for example. When semantic units between which there hold paradigmatic relations, are selected into syntagmatic chains, for example in the form of oral speech, then it is clear that they are-already by rules or grammar-subject to hierarchical relations governed by the dominant of the discourse. The latter was famously defined by R. Jakobson:

"The dominant may be defined as the focusing component of a work of art: it rules, determines, and transforms the remaining components. It is the dominant which guarantees the integrity of the structure. The dominant specifies the work" (Jakobson 1981, 751).

Naturally, this does not go only for artistic production, but is essential for all semiotic phenomena, be they complex compounds of texts or any other individually standing units. Therefore, speaking of equal relations between semiotic units arranged into chains in messages is a semiotic impossibility and deprives those units of any meaning whatsoever, taking them back to the original pool of Firstness or the sphere of mere semiotic potentiality. Heterarchic ideology has been proposed to connect biosemiotics, cognitive semiotics, and cultural semiotics for a unified description of semiosis (Bruni 2015), but this seems to be an exaggerated attempt to solve a non-existent problem, since the diverse areas where semiosis takes place are connected anyway. The whole logic of interconnected modelling systems of diverse faculties and levels (primary, secondary, tertiary modelling systems) is based on the unity of Umwelt and the semiosphere, but that unity is formed exactly on the grounds of the hierarchical organisation of semiosis.

Ideas concerning Complex Semiotic Heterarchic Theory, which is equalised with Complex Dynamic Systems Theory, try to break in through an open door by suggesting that translanguaging is an innovation allowing to move "into de-privileging of language toward conceptions of social semiotic repertoires of meaning-making and communication" and to "de-center language through a more holistic understanding of semiotic modes or resources" (Sherris, Adami 2019, 4). In semiotics and also more specifically in language studies, it is a long-acknowledged truth that language can be studied through speech behaviour, which is always unique and individual in its contextuality. Language in use makes language immanently a dynamic and versatile phenomenon, and as such-it is always social. Additionally, recalling what was said above, when talking about linguistic coding, we must not think only about natural language, but also of other language-based semiotic systems. That means-natural language has always had its place as but just one means of encoding messages in the row of other semiotic systems. Yet it depends on the contextual circumstances what weight natural language or other semiotic systems have in the particular case of any act of communication, and the semiotic value of a dominant semiotic system is decisive also for the metalevel interpretation of messages and meanings. In this sense, although principally equal, in each communicative act, semiotic systems are valued potentially differently and therefore they do form semiotic hierarchies that are inevitable for the production of any meaning as based on operations of selection. Meaning-making is an act of modelling, and modelling is concerned about the segmentation of reality into meaningful versions through selection, and selection is inevitably a procedure involving hierarchisation. There is no other way to conceive the production of meaning as opposed to the realm of merely potential meaningfulness.

\section{MODELLING IS ALWAYS DYNAMIC}

Language-whether natural language or any secondary or tertiary sign system-is thus a dynamic system that is activated in a particular context by particular communicative agents according to their particular semiotic luggage and knowledge. The dynamism of semiotic languages or sign systems has to be comprehended even more widely. As mentioned above, in order to strive towards maximum probability of the arrival of the intended meaning, messages are mostly encoded redundantly, and oftentimes this means that messages are overcoded simultaneously in several sign systems. This kind of inherent multimodality of messages holds both for texts in only natural language (for example, irony can be simultaneously assertion of truthful facts), and for texts encoded in several sign systems simultaneously. Mutual understanding of communication partners thus depends much on how big their share of understanding the diverse techniques of encoding, decoding and correcting semiotic strings forming messages is. Besides following regularities of these techniques in one prevalent sign language (for example, when reading a book or even newspaper article), communication partners preferably ought to recognise the diversity and specificity of sign systems engaged in the transmission of more complex messages (for example, when visiting theatre or asking for guidance in a foreign city). They have to be competent in using diverse sign systems and they have to be able to discriminate between those dissimilar semiotic languages in order to understand the dynamism of communication entailed by redundancy. That is, to be successful in mutual understanding, people must be quite continuously operate with very diverse codes-they must be capable of code-meshing and code-switching. 
The versatility of sign systems is certainly not an observation to be associated with translanguaging or any other possible fancyful novel propositions for studying those systems. It must even not be stressed, but justly remembered that the processual nature of meaning-making has, amongst other truths, been regarded in semiotics in the topic of code-switching at least by the following aspects. Without hereby going into nuances about vocabulary (language, semiotic system, coding system, sign system, etc.), we can see code-switching as taking place between a) natural languages, b) different language or code systems (body language, film language), c) different modalities in terms of modalisation of the intention(ality) of the message, d) different modalities in terms of using several sign systems in parallel (for example, verbal and nonverbal), e) different modes of communicating as concerning elaborated or restricted codes. There are probably additional aspects and even types of code-switching. A common feature for the majority, if not for all of them, is that whilst translanguaging is about language management that largely is being dealt with from the viewpoint of code-switching, and if languages can be compared to codes, then translanguaging is (about) code-switching. It is quite a common practice, noticed a while ago, that messages can be encoded in different sign systems, and transmitted in this manner simultaneously in terms of being multimodal and multimedial. This has been long considered in the semiotic theorising, and even a specific field of study under the name of the semiotics of code was created for the latter (represented, most notably, by U. Eco, R. Jakobson, J. Lotman); and it is hard to imagine there could be any other modality or mediality than the semiotic ones.

\section{CONCLUSION}

Translanguaging seems to be an attempt to set up a transdisciplinary research perspective based largely on multimodal analysis. It must be stressed that studies in translanguaging have two basic sides: analysis of concrete objects and metalinguistic issues. There must be no doubt in the value of the contribution case studies bring, amongst other fields, to applied semiotics. On the other hand, there apparently lie certain dangers in the metalanguage and its pretensions that lead up to the establishment of a seemingly novel research area. Taking a view from the semiotic standpoint, those metalinguistic issues are largely confusing and even misleading, since the relevant argumentation but repeats basic semiotic truths. The interpretation of multimodal analysis in translanguaging, and possibly in all multimodal analysis, coincides with the semiotic understaning of simultaneous and synoptic study of meaningful structures and processes in the syntactic, semantic, and pragmatic dimensions as these research aspects have been long laid out by the classic(s) of semiotics. It is natural that in the age of transdisciplinarity, diverse paradigms, methodologies, vocabularies blend. Likewise it seems to be expected that disciplinary boundaries disappear for the sake of centering at objects of study, not at affordancies of study. However, our above examples seem to entail a threat of not eradicating those boundaries, but blurring them. Transdisciplinary efforts thus seem to have to focus more at not mere borrowing or so-to-speak google-translating specific traditional disciplinary lexicons and toolkits. Otherwise we will find ourselves not only in terminological and methodological chaos, where we find a question to be answered: are we living in the light or twilight of novel vocabularies? Even more importantly, such a metalinguistic confusion will inevitably bring along also turmoil at the definition and description of the very objects of analysis. Instead, we should strive at compiling a unified faculty and encyclopedia of study for a unified object-world that was exactly the task taken by the establishment of Unified Science a century ago, keeping in mind the semiotic resources notified even then.

\section{REFERENCES}

Adami, E., 2019. Multimodal Sign-Making in Today's Diversity: The Case of Leeds Kirkgate Market. In: Sherris, A., Adami, E. (Eds.), Making Signs, Translanguaging Ethnographies: Exploring Urban, Rural and Educational Spaces. (= Encounters 12). Bristol and Blue Ridge Summit: Multilingual Matters, pp. 36-54.

Bezemer, J., Kress, G., 2019. Foreword. In: Sherri, A., Adami, E. (Eds.), Making Signs, Translanguaging Ethnographies: Exploring Urban, Rural and Educational Spaces. (= Encounters 12). Bristol and Blue Ridge Summit: Multilingual Matters, $x v-x v i i$.

Bernstein, B., 1964. Elaborated and Restricted Codes: Their Social Origins and Some Consequences. American Anthropologist, 66(6), Part 2: The Ethnography of Communication, 55-69.

Blommaert, J. et al., 2019. Communicating Beyond Diversity: A Bricolage of Ideas. Edited by Elisabetta Adami and Ari Sherris. In: Sherris, A., Adami, E. (Eds.), Making Signs, Translanguaging Ethnographies: Exploring Urban, Rural and Educational Spaces. (= Encounters 12). Bristol and Blue Ridge Summit: Multilingual Matters, pp. 9-35.

Bruni, L. E., 2015. Heterarchical Semiosis: From Signal Transduction to Narrative Intelligibility. In: Trifonas, P. P. (Ed.), International Handbook of Semiotics. Toronto, Ontario: Springer, pp. 1079-1097.

Cobley, P., Randviir, A., 2009. Introduction: What is sociosemiotics? Semiotica: Journal of the International Association for Semiotic Studies, 173, 1-39.

Eco, U., 1976. A Theory of Semiotics. (=Advances in Semiotics). Bloomington, London: Indiana University Press.

Jakobson, R. 1981. The Dominant. Selected Writings. Vol.3. Poetry of Grammar and Grammar of Poetry. The Hague, Paris, New York: Mouton Publishers, $751-756$.

Dusi, N., 2015. Intersemiotic Translation: Theories, 
Problems, Analysis. Semiotica 206, 181-205.

Fiske, J., 1994. Redundancy. In: Tim O’Sullivan et al., Key Concepts in Communication and Culture Studies. 2nd edition. (= Studies in Culture and Communication). London and New York: Routledge, 259-261.

Kress, G., 2001. Sociolinguistics and Social Semiotics. In: Cobley, P. (Ed.), The Routledge Companion to Semiotics and Linguistics. London and New York: Routledge, 66-82.

Kress, G., 2010. Multimodality: A Social Semiotic Approach to Contemporary Communication. London and New York: Routledge.

Lewis, G., Jones, B., Baker, C., 2012. Translanguaging: Origins and Development from School to Street and Beyond. Educational Research and Evaluation 18(7), 641-654.

Morris, Ch. W. 1938. Foundations of the Theory of Signs. (= International Encyclopedia of Unified Science 1/2 Chicago: The University of Chicago Press.

Morris, Ch. W. 1971. Writings on the General Theory of Signs. (= Approaches to Semiotics 16). The Hague and Paris: Mouton.
Nauta, D., Jr. 1972. The Meaning of Information. (= Approaches to Semiotics 20.) The Hague and Paris: Mouton.

Peirce, Ch. S., 1931-1958. Collected Papers of Charles Sanders Peirce. Vols 1-6, ed. by Hartshorne, C. and Weiss, P., 1931-1935; vols. 7-8 ed. by Burks, A. W., 1938. Cambridge, MA: Harvard University Press. (Cited as CP, ref. to volume and paragraph numbers).

Saussure, F. de, 1959. Course in General Linguistics. Bally, Ch., Albert Sechehaye, A. (Eds.). Translated from the French by Wade Baskin. New York: Philosophical Library.

Sherris, A., Adami, E., 2019. Unifying Entanglements and Dynamic Relationalities: An Introduction. In: Sherris A., Adami, E. (Eds.), Making Signs, Translanguaging Ethnographies: Exploring Urban, Rural and Educational Spaces. (= Encounters 12). Bristol and Blue Ridge Summit: Multilingual Matters, pp. 1-8.

Sonesson, G., 2014. Translation and Other Acts of Meaning: In Between Cognitive Semiotics and Semiotics. Cognitive Semiotics, 7(2), 249-280.

Torop, P., 2000. Intersemiosis and Intersemiotic Translation. European Journal for Semiotic Studies, 12(1), 71-100. 